\section{Psicología IBEROAMERICANA}

\section{Psicología Iberoamericana}

ISSN: 1405-0943

psicología.iberoamericana@uia.mx

Universidad Iberoamericana, Ciudad de

México

México

Yáñez Castañeda, Karina; Rocha Sánchez, Tania Esmeralda

Experiencias y significados de la infidelidad femenina

Psicología Iberoamericana, vol. 22, núm. 2, julio-diciembre, 2014, pp. 25-35

Universidad Iberoamericana, Ciudad de México

Distrito Federal, México

Disponible en: http://www.redalyc.org/articulo.oa?id=133938134004

- Cómo citar el artículo

Número completo

- Más información del artículo

- Página de la revista en redalyc.org

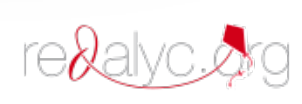

Sistema de Información Científica

Red de Revistas Científicas de América Latina, el Caribe, España y Portugal Proyecto académico sin fines de lucro, desarrollado bajo la iniciativa de acceso abierto 


\title{
Experiencias y significados de la infidelidad femenina
}

\author{
Experiences and Meanings of Female Infidelity
}

\author{
Karina Yáñez Castañeda* \\ Tania Esmeralda Rocha Sánchez \\ FACULTAD de Psicología \\ Universidad Nacional Autónoma de México
}

\section{RESUMEN}

La infidelidad entendida como el involucramiento sexual o emocional con una persona que no es la pareja ha sido pensada en forma primordial como un acto naturalmente masculino. Aunque se percibe un aumento en las cifras de infidelidad femenina, éstas se enmarcan en prejuicios y estereotipos de género, que sesgan su investigación. Así, el objetivo del presente estudio fue analizar las experiencias de infidelidad femenina considerando la influencia de las construcciones de género en ellas. Se realizaron 10 entrevistas semiestructuradas a mujeres con diversas condiciones sociodemográficas, que fueron interpretadas a través del Análisis Crítico del Discurso. Los resultados muestran que las vivencias de infidelidad conjuntan elementos psicológicos y socioculturales y, a su vez, producen reflexiones en torno a la validez de los mandatos de género que permean dichas vivencias.

Palabras clave: infidelidad femenina, categoría experiencia, mujeres, género.

\section{ABSTRACT}

Infidelity, understood as the sexual or emotional involvement with someone who is not your partner, has mainly been considered as a characteristically masculine trait. Although female infidelity is perceived to be becoming more common, the figures are affected by gender prejudices and stereotypes, adding bias to research. Therefore, the objective of this research was to analyze the experiences of female infidelity, considering how they are influenced by gender constructions. Ten semistructured interviews were carried out with women in various socio-demographic situations, and these were interpreted through the Critical Discourse Analysis. The results show that experiences of infidelity combine with psychological and sociocultural elements to stimulate reflections on the validity of gender influences that permeate these experiences.

Keywords: Female infidelity, Experience Type, Women, Gender.

Recibido: 8 de abril de 2013 - Aceptado: 30 de agosto de 2014

*Para correspondencia: karina-yanez@live.com.mx 


\section{INTRODUCCIÓN}

El debate teórico sobre si el ser humano es monógamo o polígamo es amplio. Sin embargo, varios estudios señalan la tendencia de los seres humanos para tener varias parejas sexuales a lo largo de la vida (Buss, 2003; Shackelford, Schmitt \& Buss, 2005; Schmitt, 2010). Algunas teorías sustentan la infidelidad como una cuestión biológica (Buss, 1994; Manzo, García, Pérez \& Hernández, 2009; Vargas \& Ibáñez, 2005), mientras que otras teorías dan cuenta de los pactos sociales y culturales que regulan la sexualidad de las personas (Hamilton \& Armstrong, 2009; Holmes \& Douglas, 2012; Rudman, Fetterolf \& Sánchez, 2013). Sin embargo, pese a ser una tendencia humana, existe un comportamiento diferente en función de si se es hombre o mujer, de tal suerte que sea cual sea la corriente teórica que explique la infidelidad, hay una tendencia a señalar la infidelidad masculina como un hecho natural.

Si bien la revisión llevada a cabo por Blow y Hartnett (2005) concluyó que son los varones casados quienes más parecen involucrarse en relaciones de infidelidad, especialmente cuando se trata de infidelidad sexual -dicha prevalencia también fue encontrada en algunos países de Latinoamérica como Chile, Ecuador, Brasil y México (GDA, 2010)-, un estudio realizado por Whisman y Snyder en 2007, en el que entrevistaron alrededor de 4884 mujeres, encontró que aproximadamente $6 \%$ de ellas admitió haber ejecutado una vivencia de infidelidad en el marco de sus relaciones conyugales, de manera que la infidelidad ocurre independientemente de si se es hombre o mujer y parece tener sentido sólo en relaciones donde la monogamia se considera como la única forma de parentesco válido (Labaca, 2009; Urbanik, 2012). Como ejemplo, las relaciones matrimoniales surgieron como una construcción de orden social, cuyo fin era legitimar las relaciones de parentesco entre un hombre y una mujer, estableciendo la exclusividad sexual de la mujer como un medio de asegurar la herencia a los hijos, para lo cual no era necesaria la fidelidad del hombre (Engels, 1970).

En ese sentido, como lo señalan Hernández y Pérez (2007), en los acuerdos respecto a la vivencia de las relaciones extradiádicas se hace evidente el orden genérico, pues en diferentes sociedades contrasta la prevalencia de sanciones concretas o simbólicas para las mujeres, mientras que en el caso de los hombres esta vivencia es premiada (Fernández, 2011; Therborn, 2007). De acuerdo con Rathus, Nevid y Fichner-Rathus (2005), los varones suelen contar con mayor libertad social para involucrarse en aventuras sexuales, pues existe una cultura patriarcal que así lo sustenta, en tanto que en el caso concreto de las mujeres los procesos de socialización inciden en una subjetividad ligada al deseo de vivirse y ser para otros (Basaglia, 1983; Lagarde, 1996; 2012), subjetividad que está altamente relacionada con la forma en la que viven su sexualidad y por ende, con el menor involucramiento -al menos teórico- en experiencias de infidelidad.

De acuerdo con investigaciones existentes, la infidelidad puede deberse a múltiples y diversas causas: la lucha de poder y el estatus entre los miembros de la pareja, problemas de comunicación, manejo inadecuado de celos, pérdida de la intimidad e incluso la presencia o ausencia de hijos (Blow \& Hartnett, 2005; Lammers, Stoker, Jordan, Pollman \& Stapel, 2011; García-Méndez, Rivera-Aragón \& Díaz-Loving, 2011) y no sólo se da en medio del conflicto, también existen personas cuyas características personales los llevan a tener actitudes más positivas hacia la infidelidad, mayor transgresión de las normas convencionales e incluso una imperante necesidad de emociones nuevas (Figueredo, Vázquez, Brumbach \& Schneider, 2007; Kirsner, Figueredo \& Jacobs, 2009; Zuckerman, 2007).

Por otra parte, dentro de las diferentes manifestaciones de la infidelidad existen dos formas predominantes: emocional y sexual (Hertlein, Wetchler \& Piercy, 2005; Shackelford \& Buss, 1997; Whitty \& Quigley, 2008). De acuerdo con Shackelford, Le Blanc y Drass (2000), mientras que la infidelidad sexual alude directamente a un involucramiento sexual con o sin consentimiento de la pareja primaria, el aspecto emocional se refiere más al involucramiento afectivo que se refleja, entre otros aspectos, por el tiempo y atención dedicada a la otra persona.

Dichas esferas están divididas en coincidencia con las construcciones de género que existen en torno a las características y comportamientos que diferencian a los hombres y las mujeres; de acuerdo con los estereotipos de género hegemónicos, la idea de lo "femenino" se encuentra enraizada con una exacerbación del aspecto emocional, y exalta comportamientos dulces, 
sentimentales y afectivos, aunado a una posición subordinada y de sumisión en el marco de la relación "femenino-masculino", además se pone particular énfasis en la belleza física, mientras que en la construcción de lo "masculino" predominan aspectos como la rudeza, la fuerza, la intelectualidad, la protección, la independencia, la valentía y la hipersexualidad (Acuña \& Bruner, 2001; Araujo \& Rogers, 2000; Azaola, 2009; Delgado, Novoa \& Bustos, 1998; Lagarde, 2012; Tena, 2012), en tal forma que la infidelidad sexual se asocia predominantemente con los hombres, mientras que la infidelidad emocional se asocia con las mujeres, y se reitera así la lógica dicotómica y excluyente, donde la esfera emocional parece contraponerse a la esfera sexual.

De acuerdo con los estudios de Díaz-Guerrero (1986, 2003), en la cultura mexicana el tradicionalismo familiar refuerza en las mujeres estereotipos de género asociados con la fidelidad, y aparece como una norma implícita que aún hay que cumplir (Alarcón, 2005; Díaz-Guerrero, 2003; Reyes \& Díaz-Loving, 2012); así, se vigila con más atención en mujeres que en hombres, para lo cual se auxilian de mecanismos sociales y culturales que controlan y regulan las vivencias de las mujeres. Por ello, las relaciones de infidelidad que las mujeres establecen permanecen ocultas, por lo general, lo que hace creer que la infidelidad femenina es un fenómeno que no ocurre y se cataloga de inmediato como "malas mujeres" a aquellas que lo hacen evidente, como una forma de castigo social ante la desobediencia de un rol impuesto y que, en definitiva, no necesariamente responde a los papeles ejecutados en la vida cotidiana.

En la actualidad, las personas parecen presentar características y comportamientos híbridos que superan la visión bipolar y dicotómica hombre-mujer (DíazLoving, Rocha, \& Rivera, 2004; Fernández, Quiroga, Del Olmo \& Rodríguez, 2007; Lippa, 2005; 2001; LópezZafra \& García-Retamero, 2012); asimismo, existe una constante transformación de las prácticas sociales y de los acuerdos de género convencionales, que permiten mayor libertad a las mujeres y, en consecuencia, conforman identidades, cuerpos y sexualidades en movimiento (López, 2009; Masters, Casey, Wells \& Morrison, 2013; Wilkins \& Dalessandro, 2013).
Cabe considerar que tanto el discurso social predominante como las prácticas socioculturales y de socialización, no afectan por igual a todas las personas. Tal como lo señalan Cuvi-Sánchez y Martínez-Flores (1994), en el marco de las vivencias que las mujeres tienen sobre su sexualidad y sus cuerpos, es necesario considerar también las prácticas individuales, la posición social, sus edades y sus historias personales, pues todos estos factores en conjunto introducen matices importantes en las actitudes de las mujeres y en las formas en que ellas valoran y viven la sexualidad, e incluso inciden en el grado en que el discurso hegemónico se acepta y obedece.

En consecuencia, el estudio de la infidelidad femenina advierte la necesidad de tener en cuenta la forma en la cual las construcciones de género impactan de múltiples formas los arreglos y vivencias actuales en el marco de las relaciones de pareja, no sólo considerando los cambios que cuestionan y transgreden el orden de género, sino también atendiendo el cambio que hay en torno a las expectativas sobre las relaciones de pareja, el amor, el matrimonio así como el cambio acerca del ejercicio de la sexualidad misma, pues como se dijo previamente, el concepto de infidelidad se estructura con base en los acuerdos socioculturales, según los cuales se construyen las parejas antes o después de la vida conyugal, acuerdos que se establecen en forma diferencial de acuerdo con el género asignado $y$, por ende, marcan diferencias en los procesos de infidelidad $y$ en el significado que se tiene de dicha experiencia.

A partir de lo anterior, el objetivo del presente estudio fue dar cuenta, en voz de las mujeres, de cuáles son los significados y vivencias en torno a las experiencias de infidelidad siendo ellas las protagonistas. Para lograr tal propósito se tuvo en consideración ejes críticos como las construcciones de género que permean las vivencias de las mujeres, las historias individuales de cada participante y los significados otorgados a los constructos de pareja y amor.

\section{MÉTODO}

La necesidad de considerar la vivencia de la infidelidad con las mujeres como protagonistas, hizo necesario partir de un marco interpretativo feminista y fenomenológico, con base en el cual no sólo se tendrá en 
consideración la experiencia subjetiva de las participantes, sino que se integrará el impacto que los procesos socioculturales vinculados con el género tienen en el marco de estas vivencias. Para lograr dicho objetivo, se realizó una convocatoria desde redes sociales, solicitando la participación voluntaria y anónima de aquellas mujeres que asumieran ser o haber sido infieles. Dada la dificultad para acceder a la muestra, en particular por el ocultamiento que parece caracterizar tales vivencias en el grupo de mujeres, fue necesario recurrir a un muestreo por conveniencia (Ito \& Vargas, 2005). En total, participaron 10 mujeres cuyas características se detallan en la tabla 1.

De inicio, se contactó a las participantes y tras explicarles el propósito de la investigación, se les invitó a participar. Los datos fueron obtenidos a través de entrevistas semiestructuradas de una sola sesión; las entrevistas se llevaron a cabo en aulas de clase, cafeterías y algunas en casa de las participantes. Se comenzó por llenar una ficha sociodemográfica, además de la lectura y firma de un consentimiento informado, donde se solicitó su autorización para grabar las entrevistas en formato de audio, garantizando el anonimato y confidencialidad de las grabaciones.

Posteriormente, la guía de entrevista contó con cuatro ejes de análisis: conceptuación de la infidelidad, caracterización de la infidelidad, significación de la infidelidad y construcciones identitarias en torno al género.

Para analizar la información se hizo uso del análisis crítico del discurso (ACD), considerando que los relatos de las participantes y sus experiencias mismas constituyen y a la vez son constituidas por construcciones socioculturales. Tal como lo refiere Franquesa (2002), el ACD es una herramienta que considera el contexto donde se desarrollan las experiencias, de tal suerte que a través de este tipo de análisis es factible dar cuenta de la interrelación de la experiencia con los aspectos de orden estructural.

Tabla 1. Descripción de las participantes

\begin{tabular}{|c|c|c|c|c|c|c|}
\hline Seudónimo & Edad & Tipo de unión & $\begin{array}{l}\text { Núm. de } \\
\text { hijo(a)s }\end{array}$ & Escolaridad & Ocupación & Lugar de origen \\
\hline María & 37 & Casada & 2 & Bachillerato & Ama de casa & Estado de México \\
\hline Kary & 46 & Unión libre & 1 & $\begin{array}{c}\text { Doctorado (en } \\
\text { curso) }\end{array}$ & Psicóloga clínica & Distrito Federal \\
\hline Yamilet & 31 & Casada & 2 & Bachillerato & Empleada & Estado de México \\
\hline Diana & 33 & Soltera & - & Doctorado & Docente & Distrito Federal \\
\hline Mara & 40 & Unión libre & - & Licenciatura & Empresaria & Guadalajara \\
\hline Rebecca & 29 & Unión libre & - & Licenciatura & Empresaria & Distrito Federal \\
\hline Magdala & 30 & Soltera & - & Doctorado & $\begin{array}{l}\text { Investigadora y } \\
\text { docente }\end{array}$ & Distrito Federal \\
\hline Yazmín & 23 & Soltera & - & Licenciatura & $\begin{array}{l}\text { Tesista y empleada } \\
\text { medio tiempo }\end{array}$ & Distrito Federal \\
\hline Karla & 28 & Soltera & - & $\begin{array}{l}\text { Licenciatura } \\
\text { trunca }\end{array}$ & $\begin{array}{c}\text { Asesora de bienes } \\
\text { raíces }\end{array}$ & Estado de México \\
\hline Marylú & 26 & Soltera & 1 & $\begin{array}{c}\text { Licenciatura en } \\
\text { curso }\end{array}$ & Estudiante & Estado de México \\
\hline
\end{tabular}




\section{RESULTADOS}

\section{La necesidad de sentirse amadas}

Al explorar cómo es que las mujeres explican las vivencias de infidelidad, en este estudio se encontró que suele reproducirse el discurso hegemónico que afirma la importancia de las emociones en la vida de las mujeres, de manera que en sus relatos se acentúa la presencia de sentimientos, especialmente aquellos que apelan al "amor romántico", insertos en el marco de la dinámica de pareja, de cómo se sienten y cómo les gustaría sentirse. Las participantes que se encuentran en una relación "conyugal", sea por matrimonio o unión libre, aluden con frecuencia a los sentimientos de insatisfacción que experimentan fundamentalmente por la percepción de descuido y desamor por parte de sus parejas, como lo relata Kary:

... yo creo que para todas las mujeres, pero para mí es esencial el detalle, este, pos tú sabes, ¿̇no? Que te digan que estás bonita, que, que eres lo máximo, que, ese tipo de cosas que, que se dan en el noviazgo y que poco a poco se van perdiendo en el matrimonio, yo este, como que he tratado de aceptarlo y decir, ¡bueno!, ps' es que es algo normal, ¿no?... (Kary, 46 años, unión libre, profesionista).

Esta necesidad de sentirse cortejadas, conquistadas, más en un plano sentimental o emocional, responde a las formas de amor en que las relaciones de pareja han sido construidas en la cultura mexicana y al mismo tiempo, da cuenta de la manera en que las mujeres se piensan y viven en el marco de las relaciones heterosexuales, por ejemplo, que para sentirse amadas tienen que sentirse cortejadas y conquistadas, lo cual aparentemente se busca y encuentra -de manera consciente o inconsciente- en el marco de las vivencias de infidelidad. Así, María cuenta (refiriéndose a los mensajes de texto que le envía la otra persona): “... son este, ‘ ¿cómo estás?' 'Bien, ¿y tú?' 'También', pero cosas bonitas, ¿no? Que este, 'corazón', no sé, cosas, cosas que dices: ' $¡ a y !$ ', pues como que te hacen el día, ¿no?...” (María, 37 años, casada, dos hijos).

En el discurso de las participantes aparece una subjetividad trazada en el "ser y vivirse para otros", de manera que los otros, varones, aparecen como una figura que las provee de identidad. De acuerdo con Basaglia (1983) y Lagarde (1996; 2012), la construcción de mujeres como seres para otros, en especial, según la noción de esposa (o pareja) conlleva anteponer los deseos de los demás a los propios, lo cual eventualmente produce en ellas sentimientos como la tristeza y la sensación de ser poco valoradas. De nuevo, es pertinente aclarar que no todas las mujeres que participaron en este estudio son esposas o madres; sin embargo, es cierto que la mayoría de las experiencias relatadas responde a lo que ellas nombran como carencias en su relación de pareja, y aunque las dinámicas en las parejas pueden ser muy diversas y cambiantes a lo largo del tiempo, llama la atención este señalamiento de sentir que hay un descuido por parte de sus parejas hacia ellas, que en muchos casos se asume como un devenir "normal" en toda relación y, por tanto, adoptan una postura de aparente resignación ante esa circunstancia.

Es interesante señalar que en el marco de la vida cotidiana de las dos participantes casadas, ellas precisan que parte importante de su tiempo gira en torno a las tareas domésticas y el cuidado de los hijos, ocupando en ello la mayor parte de su día y es por esa razón que relatan limitantes en la posibilidad de vivir una experiencia de infidelidad, no sólo en el sentido del tiempo y espacio que dedican a efectuar las labores del hogar, sino también en un sentido simbólico, ya que justamente al ser "madres" o "esposas" la infidelidad no tiene cabida, en particular, por las connotaciones y restricciones que esa función impone. De igual manera, hablando del espacio físico, en la literatura se ha señalado previamente el carácter de aislamiento social que encierran dichos papeles (Careaga, 2003; Durán, 2013; Maffía, 2007). Por ejemplo, Yamilet relata:

... sí se ve de otra manera porque a lo mejor estando en tu casa, tú ni siquiera te lo imaginas, ¿no?; o sea, igual te hace falta algo o discutes o de repente estás triste porque no te peló, lo que sea, pero no lo piensas, porque no lo tienes a la mano como una opción, ¿¿no? $\mathrm{Al}$ momento de estar acá y tenerlo a la mano como una opción es como empiezas a pensarlo, es como empiezas a decir “¡ay, a poco sí!”, ¿ ¿no?... (Yamilet, 31 años, casada, dos hijas). 
De esta manera, en el marco de un trabajo o actividad remunerada, las participantes emergen como sujetos de su vida (de sus cuerpos, de sus decisiones, de sus deseos, etc.), transgrediendo de una y otra manera las normas socioculturales que encubren la función de esposa y madre (en aquellas que lo son). Así, las participantes establecen una dinámica encaminada al beneficio personal, de manera que en su discurso aparecen las relaciones de amistad que ocurren dentro del espacio laboral, que parecen vivirse desde una posición de mayor libertad, donde contrario a lo que pasa con algunas otras relaciones reguladas socialmente-como la pareja, la familia o las relaciones laborales-, en éstas se establecen vínculos de intimidad y comprensión, de apertura para externar lo que se desea y lo que se piensa, haciendo contacto con un deseo propio y no con lo dictado por una función, lo que da lugar al surgimiento de vivencias de infidelidad. Por ejemplo, Yamilet relata:

... cuando entré a trabajar, la verdad es que estando en la casa con las niñas, o yéndote a tomar el café con tu amiga no es lo mismo; cuando entré a trabajar, te lo juro que se me abrieron así como que puertas, así como enormes y dices: “ $\mathrm{Q}$ ué caray!”, ¿no?, o sea, empiezas a ver tantas cosas, tantas actitudes, empiezas a conocer más tipo de gente porque pues, obviamente, en la escuela conoces a las "mamás de", nada más, o sea, no conoces más que eso... (Yamilet, 31 años, casada, empleada de una tienda de autoservicio).

\section{Experiencias infieles, ¿amor, sexo o ambos?}

$\mathrm{Al}$ explorar las distintas vivencias de infidelidad que relatan estas mujeres, se encontró la ocurrencia de esta clase de relaciones que han permanecido estables por largos periodos, experiencias que han sucedido una sola vez, así como relaciones constantes de infidelidad, lo que da cuenta de la enorme diversidad de posibilidades que configuran las experiencias de infidelidad en las participantes. Por ejemplo, María señala “... 11 años, te estoy hablando, yo voy a cumplir 21 años de casada, entonces, desde ese entonces mantengo una relación con esta persona..." (María, 37 años, casada). En tanto que Yazmín narra: “... prácticamente con todas mis parejas he sido infiel...” (Yazmín, 23 años, soltera).

Lo interesante es que, ya sea una vez o de manera repetida, varios de estos relatos aluden al involucra- miento emocional como el motivo primordial de la experiencia infiel. Sin embargo, las participantes también relatan la satisfacción de necesidades sexuales como otra motivación para ser infiel, e incluso, la relación de infidelidad permite que experimenten vivencias sexuales distintas a las que suceden con su pareja habitual. Por ejemplo, Yazmín alude a una relación sin compromisos o free: “... con él duré como un año pero igual este, pues, con él sí era meramente sexual, entonces nada más nos veíamos los fines de semana y ya, teníamos sexo, a veces comíamos, a veces no, y ya cada quien para su casa..." (Yazmín, 23 años, soltera). En el caso de Rebecca, también aparece este discurso:

... apareció esta persona porque con mi esposo nunca hubo un buen entendimiento [sexual], a la fecha no lo hay..., y obviamente con la segunda persona pues sí, sí lo hubo, entonces pues eso influyó también mucho por un lado pues [para que] me quedara ahí, este, pues sí estaba, pues estaba muy feliz y sí lo extraño mucho [risas], pero bueno, sí, no hay con mi esposo, no... (Rebecca, 29 años, unión libre, sin hijos).

Al respecto, resulta interesante que en el relato de algunas participantes, se alude a que esta búsqueda de satisfacción sexual es algo que corresponde a los varones y, por tanto, designan a sus experiencias de infidelidad como una situación masculinizada. Particularmente, Diana refiere:

... Creo que tal vez he identificado un poco que tengo como ese pensamiento muy cínico que tienen algunos hombres de decir: "eh, bueno, pues mi esposa es mi esposa, es la madre de mis hijos, es la oficial, la mujer a la que amo, la que me importa”, ésta es una vacilada, ¿no? Entonces lo que hace que no te dé culpa es el hecho de que sabes que ese amante no amenaza tu relación, ¿¿no?... (Diana, 33 años, soltera, escolaridad: doctorado).

Sin embargo, aun siendo el componente sexual el motivo por el que algunas participantes son infieles, sus narraciones también muestran una disyuntiva entre el involucramiento emocional o sexual que reproduce en algunas de ellas el amor y el sexo como un vínculo inseparable, de manera que las participantes asumen 
el ejercicio de la sexualidad como un acto que demanda la inclusión de emociones y sentimientos, especialmente amor. Por ejemplo Magdála, quien es una de las participantes que habla con mayor apertura de su sexualidad, refiere:

... una vez caí en shock porque tengo yo mi listita de hombres, ¿no?; una vez me di a la tarea de poner un corazoncito a aquellos que yo había, [por los que] había sentido algo, ¿no?, entonces de una lista de setenta $y$ tantas personas en aquel entonces con quienes me había acostado, sólo tres tenían corazón, y yo dije “ioye, qué cosa tan triste!” O sea, setenta y tres palos y sólo tres enamoramientos, o sea setenta y tres palos entre comillas, fueron varios, setenta y tres sujetos de los cuales a tres amé, qué cosa tan terrible... (Magdála, 30 años, soltera, investigadora y docente).

Lo interesante de esta participante es que, si bien, en vista de su experiencia tiene una suerte de conciencia de género que de una u otra forma le permite cuestionar algunas normas y exigencias del rol impuesto hacia las mujeres, ella subraya la importancia del vínculo emocional en las relaciones de pareja, dando cuenta del peso que los mandatos sociales pueden generar en la vida de muchas mujeres, independientemente de las experiencias de vida, tales como el acceso a oportunidades académicas o laborales, que de una u otra forma suponen un replanteamiento de los papeles convencionales y podrían asociarse a una vivencia menos tradicional.

\section{Significados de la infidelidad y procesos de transformación identitaria}

Ante la vivencia de una infidelidad, lo encontrado en este estudio apunta a que los significados son diversos. Aunque en su mayoría los significados fueron positivos en ambos sentidos -emocional y sexual-, las participantes relataron experiencias que no fueron consideradas buenas y que incluso les generaron malestares físicos y psicológicos, asociados mayormente a la educación familiar y valores recibidos por parte de sus padres, madres y hermanas. Como ejemplo, el relato de Marylú señala el malestar que le generó la experiencia de infidelidad, relacionado con lo que le enseñaron en casa: "Realmente el conflicto no fue hacia la persona, sino conmigo por los valores, ahora sí, que me inculcó mi mamá, ¿no?: el respeto, el, este, aparte del respeto, la confianza que le das a la otra persona..." (Marylú, 26 años, soltera, una hija).

Cabe señalar que a raíz de la experiencia misma de infidelidad, se pueden generar cambios en las subjetividades y construcciones identitarias de las mujeres. Por ejemplo, María señala lo significativo que fue el primer encuentro con la persona con quien mantiene una relación de infidelidad desde hace 11 años: “...en ese contacto que hubo se acabó todo, ese primer contacto fue, fue el primer contacto que tuve con él y fue... padre, fue así como que, como que ¡ay! O sea, reviví, ¿no?, me sentí bien..." (María, 37 años, casada, dos hijos).

Mara, en un ejercicio de reflexión más profundo, señala la transformación que va experimentando a partir de sus experiencias:

...a partir de que me empezó a notar distinta como que vio otra, otro foco [...] se da cuenta que yo estoy en otro canal, que me arreglo, que me veo bien, que me cuido, que ya no, este, pos soy como que, nunca he sido precisamente sí como sumisa, pero soy ahorita como que mucho más respondona, como que me vale madres o ya ni siquiera respondo, que es lo peor... (Mara, 40 años, unión libre, sin hijos).

Más allá de la estigmatización que existe ante las experiencias infieles, en especial si son mujeres las protagonistas, es innegable la posibilidad transformadora y de reflexión que se puede experimentar en el marco de estas vivencias, dando lugar por ejemplo a nuevas formas de relacionarse, tal como las mismas mujeres nombran sus experiencias: vivir un free o tener una relación de "amigovios", permitiéndose vivir dichas experiencias en un ambiente menos restringido que en el ámbito de una relación formal y acotada culturalmente; por ejemplo, dice una de ellas: “... con el free fue como darme el chance de decir 'Bueno, sí quiero esto, ¿qué?"” (Yazmín, 23 años, soltera).

Petchesky y Judd (2006) elaboraron un modelo denominado "de adaptación y resistencia" que representa un grupo de estrategias donde la conciencia de género de las mujeres produce actos que pueden ser confrontativos o conciliatorios. Así, ellas pueden em- 
plear estrategias de adaptación en las cuales eligen seguir patrones tradicionales en aras de mantener privilegios -por ejemplo, el estatus o el reconocimiento social de tener una pareja o familia-, o bien, emplear estrategias de resistencia que significan confrontaciones francas y abiertas contra un sistema opresor. Ambas estrategias representan un continuo, de modo que se hace uso de una u otra estrategia de acuerdo con el escenario. Retomando este modelo, algunas de las participantes utilizan estrategias de adaptación que les permiten mantenerse en una relación que aun cuando en la vida cotidiana ya no es la ideal, en su subjetividad sí debe ser mantenida, ya sea por presión social o por la misma construcción que ellas tienen del deber ser familiar y de pareja.

Por ejemplo, en el caso de Rebecca, quien en gran parte de su relato describe una relación de pareja monótona y aburrida, también alude a su pareja como el "ideal" de hombre, incluso en la comparación que hace con otros varones, él resulta ser una gran pareja, motivo por el cual ella permanece en esa relación:

[Refiriéndose a su pareja formal] ... siento que me quiere, siento que él ha hecho muchas cosas, siento que es una persona que está luchando realmente por mí, y de repente, por ejemplo, veo a mis primas, sobrinas, que están con personas que toman, que les pegan, que están, que las engañan, y ahí siguen y ahí siguen $y$, realmente, pues estoy con una persona bien que me valora, que me quiere, realmente no me falta nada, me, me trata muy bien, me quiere mucho, no sé, tal vez algún día pueda llegar a quererlo igual... (Rebecca, 29 años, unión libre, sin hijos).

\section{DISCUSIÓN}

Los resultados presentados permiten observar cómo convergen muchos aspectos culturales e individuales que determinan la manera en que las participantes se aproximan a los eventos de infidelidad, además de los constantes debates que surgen en los pensamientos de estas mujeres y que le dan sentido a vivir esta experiencia, por ejemplo los relacionados con los ideales de pareja. Aun con los avances que las mujeres han tenido en cuanto al ejercicio de una sexualidad más libre y encaminada a la búsqueda de placer, la presencia de un vínculo emocional sigue siendo parte importante del involucramiento sexual de las participantes y de los ideales que conforman la construcción de pareja en la actualidad (Fernández, 2004; Heilborn \& Da Silva, 2013; Lagarde, 2012).

Específicamente, el mayor aporte de este trabajo consiste en presentar la infidelidad femenina como un acto de resistencia que se asocia con los procesos de transgresión y cambio que representan nuevas posibilidades de vivirse social y subjetivamente, distanciándose en muchos casos del ideal de mujer, esposa o madre y posicionándose como sujeto de deseo. A su vez, estas estrategias de resistencia coadyuvan en el proceso de muchas de las participantes para construir identidades ligadas a una mayor independencia, libertad, y con un mayor interés por recibir un trato respetuoso y equitativo en el marco de todas sus relaciones, tanto laborales como familiares y sociales. Lo anterior parece tener un papel importante en la forma en la que transitan ante experiencias como la infidelidad, no desde el paradigma de receptoras, sino como ejecutoras de ésta.

Tal como se ha dicho en otras investigaciones (Aguilar, Valdez, González-Arratia \& González, 2013; Cunningham, 2005; Montilva, 2007), la flexibilización de roles y creencias estereotipadas en torno a los géneros y, específicamente, a los roles que se les asignan o que se esperan de ellas como mujeres, incide en que valoren y resignifiquen distintas posibilidades de vivirse y pensarse, de manera que al hablar de infidelidad femenina se observa que los mecanismos sociales que ajustan las creencias de las mujeres a lo que socialmente se espera de ellas son transgredidos en la cotidianidad, permitiendo una revalorización de aquello que se ha considerado negativo.

Lo encontrado en este estudio muestra que la infidelidad protagonizada por mujeres representa la ruptura de una cadena de prescripciones que suponía la pareja ideal según parámetros sexistas, de exclusividad y pertenencia, y revela que aunque se piense que la infidelidad acontece primordialmente en el marco de un malestar (por ejemplo, la ruptura de ideales de pareja), no es el único acontecimiento que la desencadena; existen también mujeres cuya infidelidad es un acto que representa la experimentación de cosas nuevas o distintas, en especial, dentro del marco de la sexualidad, como una de las tantas posibilidades de transfor- 
mar la manera en la que hasta ahora las mujeres se han vinculado en pareja.

Teóricamente se ha discutido en torno a la forma en que la sexualidad se cruza con el amor en el proceso de socialización de las mujeres (Giddens, 2004; Venegas, 2009; 2011) y este estudio abona a tal propuesta, pues ofrece la posibilidad de replantear que en realidad no son esferas que necesariamente están juntas pero tampoco separadas del todo, es decir, pue-

\section{REFERENCIAS}

Acuña, L. \& Bruner, C. A. (2001). Estereotipos de Masculinidad y Feminidad en México y en Estados Unidos. Interamerican Journal of Psychology, 35(1), 31-51.

Aguilar, M. D., Valdez, M. J., González-Arratia, L. N. \& González, E. S. (2013). Los roles de género de los hombres y mujeres en el México contemporáneo. Enseñanza e Investigación en Psicología, 18(2), 207-224.

Alarcón, R. (2005). Premisas histórico-socioculturales de la juventud peruana: obediencia filial y virginidad. Revista Latinoamericana de Psicología, 37(1), 81-94.

Araujo, K. \& Rogers, F. (2000). ¿El hombre existe? En A. J. Olavarría \& R. R. Parrini (Eds.). Masculinidad/ es. Identidad, sexualidad y familia. Chile: Red de Masculinidad Chile/ Universidad Academia de $\mathrm{Hu}-$ manismo Cristiano/Flacso.

Azaola, G. E. (2009). Patrones, estereotipos y violencia de género en las escuelas de educación básica en México. La ventana, 30, 7-45.

Basaglia, F. (1983). Mujer, locura y sociedad. Puebla, México: Universidad Autónoma de Puebla.

Blow, A. J. \& Hartnett, K. (2005). Infidelity in committed relationships II: a substantive review. Journal of $\mathrm{Ma}$ rital and Family Therapy, 31(2), 217-233.

Buss, D. M. (1994). The strategies of human mating. American Scientist, 82, 238-249.

Buss, D. M. (2003). The evolution of desire. Estados Unidos: Basic Books.

Careaga, P. G. (2003). El racismo y el sexismo en las expresiones sexuales. La ventana, 18, 294-310.

Cunningham, M. (2005). Gender in cohabitation and marriage: the influence of gender ideology on housework allocation over the life course. Journal of Family Issues, 26(8), 1037-1061. den pensarse como dimensiones que para algunas de las participantes están mucho más vinculadas que para otras. Dicho de otro modo, aunque pareciera que la sexualidad y la expresión de amor son polos opuestos, en la vida cotidiana de estas mujeres dichos polos se encuentran íntimamente ligados, de tal suerte que se unen y se separan a lo largo de sus relaciones "formales" y de infidelidad, poniendo en la mira la presencia de la sexualidad femenina en las relaciones de pareja. •

Cuvi-Sánchez, M. \& Martínez-Flores, A. (1994). El muro interior. Relaciones de género en el Ecuador de fines del siglo Xx. Quito, Ecuador: Ceplaes/Abya-Yala.

Delgado, G., Novoa, R. \& Bustos, O. (1998). Ni tan fuertes ni tan frágiles, resultados de un estudio sobre estereotipos y sexismo en mensajes publicitarios de televisión y educación a distancia, unicef/Pronam, México.

Díaz-Guerrero, R. (1986). Historio-sociocultura y personalidad. Definición y características de los factores de la familia mexicana. Revista de Psicología Social y Personalidad, 2(1), 13-42.

Díaz-Guerrero, R. (2003). Bajo las garras de la cultura: psicología del mexicano, 2. México: Trillas.

Díaz-Loving, R., Rocha, S. T. \& Rivera, A. S. (2004). Elaboración, validación y estandarización de un inventario para evaluar las dimensiones atributivas de instrumentalidad y expresividad. Revista Interamericana de Psicología, 38(2) 263-276.

Durán, G. M. (2013). La historia de Cira. Vivencias de una madre-esposa. Una mirada crítica al amor. Revista Venezolana de Estudios de la Mujer, 18(40), 135-146.

Engels, F. (1970). El origen de la familia. La propiedad privada y el estado. Madrid: Fundamentos.

Fernández, G. O. (2011). Las mujeres en el Islam: una aproximación. Brocar, 35, 267-286.

Fernández, R. L. (2004). Amor y sexualidad: algunos desafíos. Universidades, 28, 21-33.

Fernández, J., Quiroga, M. A., Del Olmo, I. \& Rodríguez, A. (2007). Escalas de masculinidad y feminidad: estado actual de la cuestión. Psicothema, 19(3), 357365.

Figueredo, A. J. Vázquez, G. Brumbach, B. H. \& Schneider, S. M. R. (2007) The K-Factor, covitality and perso- 
nality. A psychometric test of Life History Theory. Human Nature, 18(1) 47-73.

Franquesa, A. M. (2002). Breve reseña de la aplicación del análisis crítico del discurso a estructuras léxico-sintácticas. Onomazein, 7.

García-Méndez, M., Rivera-Aragón, S. \& Díaz-Loving, R. (2011). La cultura, el poder y los patrones de interacción vinculados a la infidelidad. Revista Interamericana de Psicología, 45(3), 429-438.

Giddens, A. (2004). La transformación de la intimidad. Sexualidad, amor y erotismo en las sociedades modernas. Madrid: Cátedra.

Grupo de Diarios de América (GDA) (2010). Hábitos sexuales del latinoamericano. Recuperado de http:// www.unifertes.com/fileadmin/user_upload/images/ Habitos_Sexuales_de_latinoamericanos.pdf

Hamilton, L. \& Armstrong, E. A. (2009). Gendered sexuality in young adulthood: double binds and flawed options. Gender \& Society, 23, 589-616.

Heilborn, M. L. \& Da Silva, C. C. (2013). Youth, gender and sexual practices in Brazil. Psicología \& Sociedade, 25(Núm. especial), 33-43.

Hernández, G. Y. \& Pérez, G. V. (2007). Un análisis feminista de la infidelidad conyugal. Nómadas, 16.

Hertlein, K.M., Wetchler, J. L. \& Piercy, F. P. (2005). Infidelity: an overview. Journal of Couple \& Relationship Theory, 4(2), 5-16.

Holmes, B. \& Douglas, K. (2012). Human nature: being clandestine. New Scientist, 214(2861).

Ito, S. M. \& Vargas, N. B. (2005). Investigación cualitativa para psicólogos. De la idea al reporte. México: Porrúa.

Kirsner, B. R., Figueredo, J. A. \& Jacobs, W. J. (2009). Structural relations among negative affect, mate value, and mating effort. Evolutionary Pshychology, 7(3), 374-397.

Labaca, Z. M. (2009). El matrimonio polígamo islámico y su repercusión en el derecho español. Revista Jurídica de Castilla y León, 18, 261-331.

Lagarde y de los Ríos, M. (1996). Los cautiverios de las mujeres: madresposas, monjas, putas, presas y locas. México: unam, Coordinación de Estudios de Posgrado.

Lagarde y de los Ríos, M. (2012). El feminismo en mi vida. Hitos, claves y topías. México: Gobierno de la Ciudad de México/Instituto de las Mujeres del Distrito Federal.
Lammers, J., Stoker, J. I., Jordan, J., Pollman, M. \& Stapel, D. A. (2011). Power increases infidelity among men and women. Psychological Science, 22(9), 1191-1197.

Lippa, R. A. (2001). On deconstructing and reconstructing masculinity femininity. Journal of Research in Personality, 35, 168-207.

Lippa, R. A. (2005). Gender, nature and nurture. Estados Unidos: LEA.

López, M. D. (2009). Transformaciones familiares y domésticas/Las mujeres protagonistas de los cambios. DemoS (11). Recuperado de http://revistas. unam.mx/index.php/dms/article/viewFile/6719/ 6239

López-Zafra, E. \& García-Retamero, R. (2012). Do gender stereotype change? The dinamic of gender stereotypes in Spain. Journal of Gender Studies, 21(2), 169-183.

Maffía, D. (2007). Epistemología feminista: la subversión semiótica de las mujeres en la ciencia. Revista Venezolana de Estudios de la Mujer, 12(28), 63-98.

Manzo, D. J., García, M. R., Pérez, P. M. \& Hernández, A. M. (2009). La selección sexual. Revista de divulgación científica y tecnológica de la Universidad Veracruzana. XXI(3). Recuperado de http://www. uv.mx/cienciahombre/revistae/vol22num3/articulos/sexual/index.html

Masters, N. T., Casey, E., Wells, E. A. \& Morrison, D. M. (2013). Sexual scripts among young heterosexually active men and women: continuity and change. Journal of Sex Research, 50(5), 409-420.

Montilva, H. M. (2007). Individualización femenina y cohabitación sin papeles: el caso de las profesionales de Santiago de Chile. Kairos, 11(20).

Petchesky, R. P. \& Judd, K. (2006). Cómo negocian las mujeres sus derechos en el mundo. Una intersección entre culturas, política y religiones. México: El Colegio de México/ Centro de Estudios de Asia y África/Programa Interdisciplinario de Estudios de la Mujer/Programa de Salud Reproductiva y Sociedad/ Grupo Internacional de Investigación y Acción sobre Derechos Reproductivos.

Rathus, S. A., Nevid, J. S. \& Fichner-Rathus, L. (2005). Human sexuality in a world of diversity. Estados Unidos: Pearson. 
Reyes, R. N. \& Díaz-Loving, R. (2012). La virginidad: ¿una decisión individual o un mandato cultural? Psicología Iberoamericana, 20(2), 33-40.

Rudman, L. A., Fetterolf, J. C \& Sánchez, D. T. (2013). What motivates the sexual double standard? More support for male versus female control theory. Personality and Social Psychology Bulletin, 39(2), 250-263.

Shackelford, T. K. \& Buss, D. M. (1997). Cues to infidelity. Personality and Social Psychology Bulletin, 23, 10341045.

Shackelford, T. K., LeBlanc, G. J. \& Drass, E. (2000). Emotional reactions to infidelity. Cognition and Emotion, 14, 643-659.

Shackelford, T. K., Schmitt, D. P. \& Buss, D. M. (2005). Universal dimensions of human mate preferences. Personality and individual preferences, 39, 447-458.

Schmitt, D.P. (2010). Human mate choice. En M. P. Muehlenbein (Ed.). Human Evolutionary Biology. Estados Unidos: Cambridge University Press.

Tena, G. O. (2012). Amando con el cuerpo: un análisis feminista de la relación sexo-género-deseo en la ciencia. En Julio Muñoz (Ed.). Totalidades y complejidades: crítica de la ciencia reduccionista. México: CEIICH-UNAM.

Therborn, G. (2007). Familias en el mundo: historia y futuro en el umbral del siglo xxi. En I. Arriagada, \& V. Aranda (Coords.). Cambio de las familias en el marco de las transformaciones globales: necesidad de políticas públicas eficaces. Santiago de Chile: Naciones Unidas/CEPAL.
Urbanik, J. (2012). Del matrimonio con el fantasma a las uniones del mismo sexo. La función jurídica y social del matrimonio. Cristianismo y mundo romano, 219230. Recuperado de http://urbanik.bio.wpia.uw.edu. pl/files/2012/05/matrimonios-fantasmas.pdf

Vargas, F. J \& Ibáñez, R. E. (2005). Problemas maritales, la infidelidad desde una perspectiva del vínculo. Revista Electrónica de Psicología Iztacala, 8(1), 107-124.

Venegas, M. (2009). ¿Cuál es el lugar de la sociología en la educación afectivosexual? La política afectivosexual. En R. Fernández, M. Centella, \& M. Chaves (Comps.). La comunidad educativa ante los resultados escolares. Barcelona: Milrazones.

Venegas, M. (2011). Un modelo sociológico para investigar las relaciones afectivosexuales. Revista Mexicana de Sociología, 73(4), 559-589.

Whisman, M. A. \& Snyder, D. K. (2007). Sexual infidelity in a national survey of American women: differences in prevalence and correlates as a function of method of assessment. Journal of Family Psycho$\log y, 21(2), 147$.

Whitty, M. T. \& Quigley, L. (2008). Emotional and sexual infidelity offline and in cyberspace. Journal of Marital and Family Therapy, 34(4), 461-468.

Wilkins, A. C. \& Dalessandro, C. (2013). Monogamy lite: cheating, college, and women. Gender \& Society, 27(5), 728-751.

Zuckerman, M. (2007). Sensation seeking and risky behavior. Estados Unidos: American Psychological Association. 